\title{
Research on the Preference of Thinking Mode and Its Teaching Strategy: A Case Study on Chinese Teaching*
}

\author{
Pei PAN
}

The No.1 Primary School Attached Wuhan University, Wuhan, Hubei, China (P126027@ 126.com)

\begin{abstract}
As a result of specific cultural and historical environment, preference of thinking mode may vary from nation to nation and individual to individual. It is reflected by the preference of divergent thinking or convergent thinking, image thinking or abstract thinking, intuitive thinking or logical thinking, creative thinking or reproductive thinking. Therefore, it is necessary to correctly grasp the dialectical relation of various thinking training in teaching procedures. In view of the above, we should take the appropriate countermeasures for improving the effectiveness of teaching and thinking training.
\end{abstract}

Keywords - thinking mode, preference, teaching, countermeasure, thinking training, dialectical relation

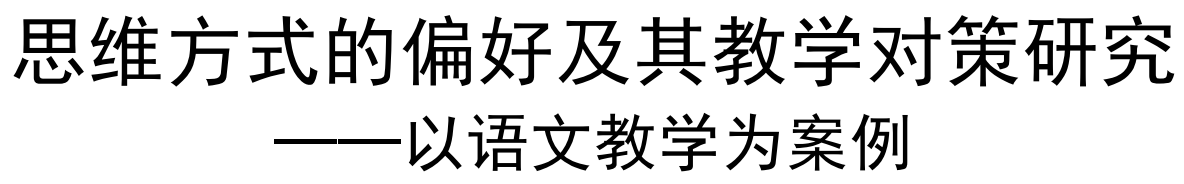

潘沛

武汉大学第一附属小学, 武汉, 湖北, 中国

摘 要 源于特定的文化环境和历史环境，不同民族、不同个人可能具有不同的思维方式偏好。这反映在对于求异思维或者求同 思维的偏好, 形象思维或者抽象思维的偏好, 直觉思维或者逻辑思维的偏好, 创造性思维或者再造性思维的偏好等, 因此, 教学过程 中需要正确地把握各类思维训练的辩证关系，据此采取相应的对策，以提高教学及思维训练的效果。

关键词 思维方式, 偏好, 教学, 对策, 思维训练, 辩证关系

\section{1. 引言}

每个正常的人都可运用多种思维方式, 其中一种或几 种思维方式相对其他思维方式或相对他人来说, 比较 “长 于运用” 或占据主导地位, 这是因为每个人的经历和社会 环境不同, 所接受的教育和文化熏陶及其个性心理特征不 同。不同的民族往往相对来说也具有其偏好的思维方式。 这就要求我们在教学中采取相应的对策。本文以语文教学 研究为案例, 此项研究在各科教学中具有普遍性。

\section{2. 求异思维或者求同思维的偏好及其教学对策}

1971 年起草 “中美上海公报” 时, 当年的美国国务卿 基辛格对周恩来总理说, 东方人的思维习惯是在 “异” 中 求 “同”, 西方人是在 “同” 中求 “异”。其实, 早在 19 世 纪, 德国唯物主义哲学家费尔巴哈就曾指出: “东方人见到
了统一忽视了差别, 西方人见到了区别遗忘了统一。 [1] 不同民族的思维方式偏好对政治、外交和社交的影响启迪 了我们对其在哲学上的了解与在相应教学对策上的思考。

根据解决问题时的思维方向, 可以把思维分为求同思 维和求异思维两类。前者是把相关的各种信息集中起来, 得出一个最好的答案; 后者是从一个目标出发, 以不同途 径寻求各种答案, 也称为发散性思维, 是创造性思维的主 导成分。两者都是智力活动不可缺少的思维。否认 “求异” 就有可能因循守旧，保守落后; 否认 “求同” 就有可能忽 视客观规律, 陷入不可知论。“求异” 和 “求同” 两种思维 形式是在对立中共存的。

既然中国人大多习惯于 “异” 中求 “同”, 就应在教学 中多加训练 “同” 中求 “异”。例如在语文阅读教学中, 教 师应该有意识地引导学生突破教材的限定范围, 突破思维

*武汉市武昌区课题支持（课题号：2012G98）, 作者是课题主持人。 
习惯的束缚, 由一点扩展发散, 多角度、多层面地思考问 题: 可以变换语言, 重新组织所阅读的文章, 引导学生领 悟该文的创作方法, 培养布局谋篇的能力; 可利用语文学 科知识结构的横向联系, 通过比较, 使学生获得整体性知 识, 对所学知识产生立体感; 对于教材中的说明文和寓言 故事, 可引导学生联系实际, 扩大其视野; 对于抒情性文 章, 应该鼓励学生的个性化阅读, 依据自身生活实践、已 有的认知水平去理解作品, 重新建构, 实现多元解读; 还 可以通过 “提供词语组成句子” 训练学生的求异思维能力, 例如, 引导学生用 “小羊”、“的”、“是”、“青草”、“吃掉” 等词语组成句子, 在学生充分发言之后, 他们会用同样的词 语组成不同的句子来表达同样的意思: “青草是小羊吃掉 的”、“小羊吃掉的是青草”、“吃掉青草的是小羊”。在这样 的练习中, 可以让学生充分发挥他们的学习积极性、主动性 进行求异思维。尤其是小学生, 正处在由无意想象向有意 想象发展的年龄阶段, 而想象本身就是一种主动的求异思 维活动, 利用这个最佳时期, 通过想象进行求异思维训练。

应用范文教学时, 如果不重视求异思维训练, 过分强 调仿写, 就容易使学生受范文的约束, 形成思维定势, 写 不出自己的真情实感, 难以掌握写作的技能。作文时不管 是写景状物, 还是叙事记人, 都可以引导学生从不同侧面 和角度来写, 描写的侧面和角度不同, 所要表达的主题也 就可能不同。

在训练学生求异思维的变通性时, 要营造一个舒畅宽 松的教学环境, 引导学生不拘常规, 寻求变异, 从多方面 探求问题的答案和方法, 得出独特见解, 这就需要教师善 于发现学生在回答问题时的闪光点, 激活学生的求异思维。 当然, 学生求异思维的结果并非都是正确的, 教师不可为 了 “随大流” 就对学生的任何不同想法全都加以肯定, 但 也不必马上粗暴地否定。教师必须引导学生自己以常识、 科学知识和逻辑规律对求异结果加以验证, 遇到问题时, 帮助学生分析求异思维中的合理因素与不合理因素。

此外, 求异思维和求同思维都不是孤立的思维形式, 一个创造思维的过程, 就是在两者的多次变换中完成的。 要开拓思路, 要培养创造精神, 不单是靠求异思维就能奏 效的, 在这过程中, 求同思维也是一种重要的、经常运用 的思维形式。在求同思维的启发下, 学生 “同中求异” 思 维才具有动力。一位专家介绍的 “互联网搜索一 “同中求 异, 作文法” 对我们的教学很有启迪。写作前, 作者先根 据实践与研究中产生的议题确定若干个关键词, 再通过关 键词在网络上进行搜索 (求同), 把搜索到的相关材料进行 䇻选、归纳、分类, 借以开拓思路, 分析出新的观点 (同中 求异), 这是文献研究法在网络时代的应用, 然后以新观点 在网络上搜索相关的依据 (异中求同), 进而以此新观点作 为科学假设组织教育实验, 这是实验研究法在网络时代的
应用, 从而验证新观点 (求异)。这是 “求同一同中求异 一一异中求同一一求异” 的辩证思维过程, 虽然这种方法 比较适合的是青年写论文, 但是, 也可以作为语文教学中 把握求异思维训练和求同思维训练辩证关系的借鉴。

\section{3. 形象思维或者抽象思维的偏好及其教学对策}

英汉翻译专家在比较不同民族的语言与其思维特点的 关系时认为: “英民族擅长于用抽象概念表达具体的事物, 比较重视抽象思维能力的运用, 而汉民族却恰恰相反, 他们 更习惯于运用形象的方法表达抽象的概念, 不太重视纯粹 意义的抽象思维。” [2] 思维差异对翻译的影响及其研究启 迪了我们在语文教学方面的思考: 汉民族文字语言的产生 和发展是汉民族 “长于形象思维” 的结果, 这种文字语言 又潜移默化地影响着汉民族下一代 “长于形象思维” 的特 点。

我国语文教学中多有偏重于形象表达的现象。对于既 可以形象表达, 也可以抽象表达的同一种意思, 教学中多 鼓励学生用形象表达, 例如, 对作文中诸如 “不入虎穴, 焉得虎子”, “快刀斩乱麻”、“井水不犯河水”, “眉毛胡子 一把抓”, “众人拾柴火焰高” 等形象表达的评价高于其相 应的抽象表达。此外, 成语、诗词或文章中, 形象表达更 受欣赏也大大地多于抽象表达, 例如, 两个不同意思的成 语 “吴牛喘月” “ “实密似疏” 出现在同一典故中, 前者 出自于古代一位臣子之口, 后者是皇帝在与他对话时的“金 口玉言”。在以后的各种文字资料中, 前者被大量地引用, 甚至还应用在日用器血中, 可见家喻户晓; 然而后者却很 少被人们引用, 甚至一些成语词典也未收录。其主要原因 是, 前者比较形象、生动、直观; 而后者比较抽象, 其寓 意更富有哲理性、思辨性。

中国人比较容易接受、欣赏和运用形象表达, 长于形 象思维。这样的思维特点并非不好, 不然, 怎么会有古代 中国的 “四大发明” 呢? 然而, 在现代科学发展中, 获得 诺贝尔自然科学类奖项的华人已有 8 位, 但他们全都不是 中国籍, 排除某些其他原因, 考虑能鞭策我们改进的因素, 应考虑人们所受教育这一因素。所受的教育包括多方面, 其中之一是: 我们的教师、教材、各种读物、高考, 甚至 成语、诗词、文章和语言等都受 “长于形象思维” 的影响, 那么学生当然也会受到 “长于形象思维” 的影响。

根据思维的凭借物和解决问题的方式对思维进行分 类, 可以从另一角度将其分成形象思维和抽象思维。形象 思维虽是发展抽象思维的基础, 并可校正抽象思维, 然而 抽象思维却可以促进、深化、规范和引导形象思维, 可将 形象思维从不自觉提高到自觉的高度。两者相得益彰, 往 往是共同参与思维活动, 具有协同性, 而且在一定条件下 
互相转化。把握形象思维与抽象思维的辩证关系, 有利于 人大脑的左右两半球都处于共同协作的有效思维之中, 这 是发展现代科学必须的。如果我们中国人在发挥 “长于形 象思维” 的基础上, 还要注重抽象思维, 将更能促使我国 现代科学事业更快速地发展。教学中如果能避免因无意识 地偏重形象思维训练, 而相对地忽视抽象思维训练, 能够 在重视形象思维训练的基础上, 还要注重抽象思维训练, 注重两者的辩证关系, 将更能促进我国语文教学的变革。

在得到正常教育的情况下, 小学中高年级学生的抽象 思维将得到迅速发展, 初中生的抽象思维将开始占主导地 位。教学中要适应儿童少年的上述思维发展规律, 要适时 地以其前期接受的形象思维训练为基础, 促使学生从形象 思维为主导向抽象思维为主导的过渡, 将两者结合起来训 练。例如, 对于小学低年级学生, 可以运用看图说话、口 头作文训练等, 有意识地把形象思维和抽象思维结合起来。

\section{4. 直觉思维或者逻辑思维的偏好及其教学对策}

根据思维的结论是否有明确的思考步骤和思维过程中 意识的清晰程度, 可以把思维分为直觉思维和逻辑思维两 类。直觉思维是指凭借直觉进行的快速、直感、顿悟性的 思维。儿童少年一般比较偏好直觉思维, 对此可以在中小 学教学中利用, 但不能迁就, 必须把握直觉思维训练和逻 辑思维训练的辩证关系。

在课堂教学中适当地借助多媒体, 有利于直觉思维训 练。在有条件的学校, 师生都乐于应用多媒体辅助教学, 不过, 多媒体的应用必须有个 “度”, 如果语文课上大量地 播放电影、图画、音乐, “喧宾夺主”, 用多媒体替代了语 言文字教学, 这就不是语文课, 而是美术课或音乐课了。 如何把握这个 “度” ? 首先是教师要明确多媒体的直觉作 用应该严格控制在 “帮助学生与语言接触” 的范围内, 要 有意识地强调这一作用。其次是在应用中, 要帮助学生从 直观形象入手, 去细淢、逼真、鲜活地理解抽象的语言文 字, 延展观察感受过程, 丰富认知感受, 充实表象储备, 内化情境训练孕育悟感; 要积极发挥多媒体信息的刺激作 用, 使学生在亲临其境的感觉中产生联想和想象, 扩大学 生的思维空间, 经过启发、分析、比较, 逐步摒弃非本质 属性, 揭示出本质属性; 要自觉地在直觉思维训练的基础 上适时地进行逻辑思维训练, 并使思想得到升华。具体操 作上, 可在预先制作好的多媒体课件中, 针对学生在多媒 体辅助教学中可能出现的各种思维火花, 制作成相应的启 发、分析、比较、摒弃和揭示等多种分支教学流程, 利用 课件的 “超级链接” 功能, 分别链接在主流程中学生可能 出现思维火花之处, 以便在教学中需要时及时地选用分支 教学流程, 避免失去把直觉思维训练与逻辑思维训练结合
起来得以升华的良机。

此外, 不可单纯依靠课内应用多媒体来模似现实, 还 需课内外结合, 注重与现实生活的结合, 教师应引导学生 观察生活、体验生活, 总结生活中所看到、听到的, 把对 生活的一些感悟和想法应用于语文学习中, 以文字抒发生 活中的真实情感; 还可有计划地安排学生观察实物、标本、 模型或小实验。要强化观察训练, 促使学生高效地调用观 察感官, 提高直感效能。

要在直觉思维训练的基础上进行逻辑思维训练, 需要 训练学生学会积累, 平时有意识地积累知识、技能和经验; 要从分析具体事例入手, 归纳出其中的道理, 并作出判断, 引导学生逐步把直觉思维与逻辑思维结合起来; 还应从直 觉思维认识过程一开始就把对象作为一个整体来观照, 避 免把直觉中的具体内容拆散开来, 避免肢解课文的整体, 避免孤立地讲一个段落、一个层次; 要善于通过干练而富 于情感的启迪导语, 触发学生的灵感, 将其带入某一特定 的问题情境之中; 教师应该点到为止, 造成一种心理上的 悬念; 要引领学生运用直觉参与逻辑思维, 优化学生头脑 中解答问题的思路。课文中有许多貌似平常的词语或段落, 但体现了一定的逻辑关系, 学生稍不注意就容易忽略, 教 师必须有意识地抓住这些词句和段落, 在直觉思维训练的 基础上, 巧妙设置疑问, 让学生从中理解其逻辑关系; 在 写景状物的课文中, 作者一般都是严格按照空间层次或部 位顺序来描述的, 学生仿写作文时如果将空间层次或部位 顺序不合理地颠倒了, 就会引起逻辑上的混乱, 要使学生 在犯错的过程中意识到这一点; 要注意收集学生在语文学 习中常出现的逻辑错误, 根据错误的性质, 分门别类, 加 以归纳, 由教师分析其中有代表性的一二例, 其他的由学 生作改错练习, 引导他们由此及彼, 触类旁通, 进行逻辑 思维训练。

逻辑思维是思维正确性的坚实基础, 而直觉思维是思 维的主要创新源泉。例如在训练口语交际能力的过程中, 如果忽视逻辑思维的指导作用, 忽视理性分析, 学生对话 语的理解就会失之偏颇; 如果忽视直觉思维的作用, 就难 以接受人文性的熏陶, 会导致主体性和人文性的缺失。整 合二者的作用, 既有对语言的感悟和体验, 又有理性分析, 在培养学生口语交际能力的同时, 使其受到人文精神的熏 陶、直觉思维训练和逻辑思维训练。

\section{5. 创造性思维或者再造性思维的偏好及其教学对策}

根据思维的创新成份及其结果的新颖程度, 可将其分 成创造性思维和再造性思维两类。近年来, 创造性思维训 练及相关研究受到应有的重视; 然而, 再造性思维训练及 其两者辩证关系的研究却相对地弱一些。

再造性思维是根据已有经验和知识来学习新知识、解 
决新问题的思维形式, 这种思维在学生理解、掌握及运用 知识过程中是必须的; 而且, 创造性思维是离不开再造性 思维的, 前者是在后者基础上发展的, 两者之间存在着相 互渗透、相互转化的动态关系。例如, 学生在阅读文艺性 课文时, 再造性想象能够使课文中用文字叙述和描写的景 物、人物和事物在学生的头脑中再现出来, 形成一幅幅的 画面, 把学生的思维带到作者所描述的意境中去, 这就是 所谓的 “如临其境、如观其状、如见其人、如闻其声”。教 师必须为学生提供与所描述形象相类似的直观材料, 使形 象的描写与生动的叙述相结合, 使其具备形成新形象有关 的知识、经验, 正确地理解关于事物的描述, 学生才有可 能获得正确的再造性想象, 同时也受到再造性思维训练, 以利于学生今后能更自觉地运用再造性思维, 更独立地获 得再造性想象。

应正确把握再造思维训练和创造思维训练的辩证关 系。例如在作文训练中, 尤其是起始阶段, 可以让学生在 写作前欣赏一篇类似的范文, 引导学生借鉴。应着眼于引 导学生学习范文在构思立意、选材、遣词造句等方面的长 处, 还可借鉴范文中怎样描绘人物性格、事件发展、自然 景物、社会环境等细节。教师要引导学生在范文中找规律, 总结归纳方法, 引导学生了解各种表达技巧, 把课堂上学 到的写作知识转化为能力, 在自己的写作中运用; 至于学 生借鉴范文具体写作时, 则只求其神似, 绝不能照抄。为 了避免因模仿范文 “限制学生的思维”, 作文训练的后期也 可以 “先练后导”, “先练” 时要放手让学生思考, “后导” 时要交给学生思维的工具; 还可改变范文中的一两个要素, 例如改变人称重写故事, 或以范文中的片段仿写, 或模仿 范文由学生续写, 鼓励创新。

老师可以通过批改作文, 引导学生从修改中反向模仿 写作中的失误。尤其是小学中低年级学生, 因阅历、知识 和能力的限制, 其思维免不了存在着一些缺陷, 教学中可 引导学生拓宽思路, 反向思维, 以反祄正, 通过思维过程 中一正一反的训练, 培养他们辩证思维, 从而使学生的思 维灵活变通, 产生新的见解。老师批改作文还要更新评价 观念, 提倡 “以我手写我心”, 鼓励学生的创新意识。

\section{6. 针对思维方式偏好的通用教学方法}

\section{1 调动不同思维方式偏好的学生积极参与协作学习}

使每个不同思维方式偏好的学生得以和谐发展的主要 方法之一是, 引导他们在协作学习中充分表现自己的智慧, 学习别人的智慧, 发展大家的智慧, 实现信息分享和思维 互补, 学会与他人合作。可以采用动态组合法, 既有同质
编组也有异质编组, 既有组内合作也有组间交流。教师要 记录不同类型学生的成长信息, 适时解释学生的思维方式 倾向及其特征的变化, 帮助学生自我调控、取长补短。

\section{2 利用思维导图改进思维训练}

在语文课文教学中画思维导图, 需要首先把课文主题 画在纸中央, 然后在主题之下延伸出次主题, 之后延伸出 下一层细节及更次一级的细节, 主题、次主题和各级细节 均可用关键词和图像表示。在完成思维导图的流程后, 用 阿拉伯数字标记其顺序, 使我们一目了然地了解整篇课文 的布局谋篇, 使教学流程更加科学、有效, 帮助师生掌握 有效的学习方法和策略。

课文教学中可以先由学生在电脑中依课文画思维导 图, 然后从局域网中调到小组的展示电脑中交流, 最后老 师从局域网中调出部分学生画的图加以评点, 并应用老师 事先准备的 PPT 软件用大屏幕讲解; 如果没有局域网系统 则先由学生在纸上画思维导图, 小组交流时教师巡视, 最 后教师用大屏幕演示分析。在此过程中, 教师要根据学生 在思维导图作品中反映的问题, 了解学生对知识的理解和 认识程度, 及时引导学生自己解答思维过程中遇到的问题。

在语文教学的思维训练中利用思维导图的可视性, 可 清晰地描绘思维的线路, 有利于使所要解决的问题逻辑清 晰、结构层次分明, 有助于改进学生的逻辑思维和创造性 思维, 有助于把形象思维和抽象思维结合起来, 激活学生 大脑中存在的图式, 使思维话动从一个分支发展到另一个 分支, 思维过程更加形象直观, 更加符合学生的年龄和心 理特点。引导学生沿着思维导图的框架进行认知, 有利于 提升学生的认知水平。利用思维导图便于不同思维方式偏 好的学生参与协作学习, 便于教师针对各类思维训练的辩 证关系采取相应对策, 因材施教。

以上限于篇幅和个人的局限, 难以面面俱到, 不过, 现在的关键是教师要具有努力把握教学中各类思维训练辩 证关系的意识。本文仅针对当前存在的主要问题提出相应 的建议, 希能为我们注重改变不正常的倾向抛磚引玉。

\section{参考文献(References)}

[1] Baolai Cai, Yinfeng Jin, "The innovation of educational theory in curriculum reform," Curriculum; Teaching Material and Method, Beijing, no.10, 2007, pp. 5-6.

[2] Haitao Zhang, "The influence of English and Chinese thinking differences on translation,"Chinese Translators Journal, Beijing, no.1, 1999, pp. 21-22. 